\title{
Aberrant cervical thymus and the role of ultrasonography: A case report
}

\author{
Emre Ünal, Hanife Gülden Düzkalır, Seray Kurt-Güney, Emek Tolga Işıldak \\ Department of Radiology, Zonguldak Atatürk State Hospital, Zonguldak, Turkey. \\ E-mail: emreunal.rad@gmail.com \\ Received: 25 May 2015, Accepted: 16 September 2015
}

\begin{abstract}
SUMMARY: Ünal E, Düzkalır HG, Kurt-Güney S, Işıldak ET. Aberrant cervical thymus and the role of ultrasonography: a case report. Turk J Pediatr 2016; 58: 230-231.

Ectopic/aberrant cervical thymic tissue is a rare cause of neck mass and usually detected incidentally. Aberrant thymic tissue can occur anywhere in the course of thymic descent from the angle of the mandible to the superior mediastinum. We report a case of aberrant cervical thymus demonstrated by ultrasound.
\end{abstract}

Key words: ectopic, thymus, ultrasound.

Neck masses are common disorders in infants and children with a wide range of differential diagnosis, including congenital or acquired in nature. The aberrant cervical thymus is an uncommon cause of neck mass usually detected incidentally. It can occur anywhere along the pathway of thymic descent (thymopharyngeal duct) from the angle of the mandible to the superior mediastinum ${ }^{1-3}$. With the advent of high-frequency ultrasound probes, it is now possible to demonstrate the similarity of echotexture between the thymus and neck mass ${ }^{4}$. Unique features of the thymus anatomy (internal echo characteristics) allows the diagnosis.

We present a case of aberrant cervical thymic tissue with the aim of drawing attention to ultrasound (US) imaging features, which may prevent further investigation.

\section{Case Report}

A four-year-old girl was referred to US section in our radiology department with a history of an atypical infrathyroidal lymph node which was detected by US in a different clinic. Medical history and physical exam were unremarkable. US examination revealed non-cystic soft tissue mass, located inferior to right lobe of the thyroid gland (Fig. 1a).The lesion showed identical echotexture to the normally located thymus gland (Fig. 1b).

\section{Discussion}

The thymus develops mainly from the ventral wing of the third pharyngeal pouch on each side and elongates inferomedially, forming the pathway called as thymopharyngeal duct/tract ${ }^{1}$. The thymopharyngeal duct is obliterated as the thymus reaches its definitive location. However, during this process a thymic remnant may occur anywhere along the cervical pathway due to failure of involution of the thymopharyngeal duct. The entire or partial thymus may also fail to descend ${ }^{1,3}$. In patients with an undescended thymus, only half or less of the thymus is present in the chest ${ }^{4}$. The latter presents as a larger ectopic neck mass.

Cervical thymus is an uncommon cause of neck mass and usually detected incidentally ${ }^{4}$. Eighty \% to $90 \%$ of these lesions remain asymptomatic. Thus, the true incidence of ectopic cervical thymus is still unclear. Diagnosis of a cervical thymus by solely US imaging is important due to lack of ionizing radiation and no need for contrast media or sedation. Also, US is the first modality of choice particularly in children. Song et al. ${ }^{2}$ reported that US appeared to be the most direct and practical imaging method for the diagnosis of aberrant cervical thymus. Internal echo characteristics of an aberrant cervical thymus can easily be demonstrated by US. Such findings are inner multiple echogenic linear structures, 

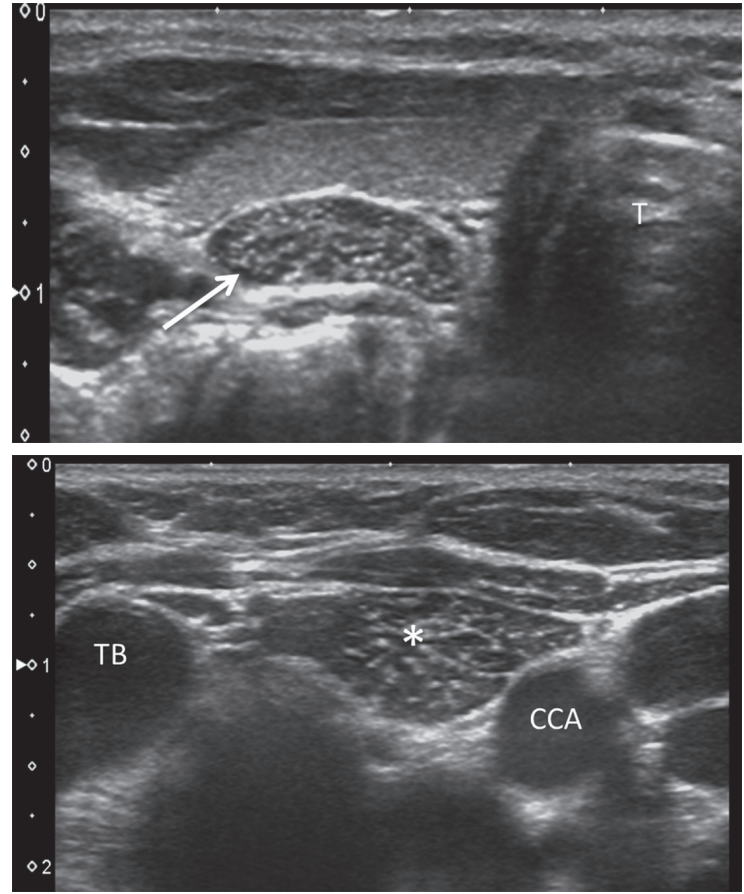

Fig. 1. Aberrant cervical thymus in a 4-year-old girl (a) US image of the right thyroid gland shows a well-defined, infrathyroidal soft tissue mass (arrow) with similar echo pattern to the mediastinal thymus. (asterisk) (b) Transverse US image at the level of upper mediastinum shows normally located thymus (asterisk). Multiple inner echogenic foci scattered throughout the parenchyma is noted.

angulated configuration and foci surrounded by hypoechoic rims which are also identical to those of the mediastinal thymus ${ }^{1,2}$.

In a study by Han et al. ${ }^{1}$ authors correlated the US and histopathological findings of the thymus anatomy and concluded that the multiple echogenic linear structures within the thymus represent connective tissue septa and accompanying blood vessels. They also reported that ultrasound characteristics of thymic tissue are unique enough to make a diagnosis of aberrant cervical thymus and biopsy may not be required for the diagnosis ${ }^{1}$. Zielke et al. ${ }^{4}$ also concluded that the mass in question is ectopic thymic tissue can be confirmed by US, depending on the unique imaging features. Thus, biopsy or surgical removal may not be required. However, large ectopic thymic masses and cervical thymic cysts may cause mass effect on adjacent structures $1,2,4$. In such cases, the diagnosis of ectopic thymus is more challenging and fine needle aspiration cytology or surgical biopsy may be reauired.
On the other hand, cases with ectopic thymic carcinoma and thymoma have also been reported in the literature ${ }^{5,6}$. However, these tumors occur more frequently in adults than in children $^{5,6}$. Also, to the best of our knowledge there is not enough data to point out that the malignant transformation rate of ectopic thymic tissue is any greater than normal thymic tissue. Thus, patients with aberrant cervical thymus could be followed without resection ${ }^{4}$.

In conclusion, aberrant cervical thymus should be considered in the differential diagnosis of asymptomatic neck masses, particularly in infants and children. Presence in the course of thymic descent and unique ultrasound features make the diagnosis of aberrant cervical thymus.

\section{REFERENCES}

1. Han BK, Yoon HK, Suh YL. Thymic ultrasound. II. Diagnosis of aberrant cervical thymus. Pediatr Radiol 2001; 31: 480-487.

2. Song I, Yoo SY, Kim JH, Hong E, Yoon HK. Aberrant cervical thymus: imaging and clinical findings in 13 children. Clin Radiol 2011; 66: 38-42.

3. Tovi F, Mares AJ. The aberrant cervical thymus. Embryology, Pathology, and clinical implications. Am J Surg 1978; 136: 631-637.

4. Zielke AM, Swischuk LE, Hernandez JA. Ectopic cervical thymic tissue: can imaging obviate biopsy and surgical removal? Pediatr Radiol 2007; 37: 1174-1177.

5. Tsukada J, Hasegawa I, Sato H, Kakefuda T, Sugiura $\mathrm{H}$, Narimatsu Y. Ectopic cervical thymoma located in the carotid triangle. Jpn J Radiol 2013; 31: 138-142.

6. Yao WT, Chen $\mathrm{CH}$, Lee JJ, Chen BF, Liu TP. Ectopic thymic carcinoma in the neck. Ann Thorac Surg 2010; 90: 666-668. 